\title{
Intrinsic Cardiac Catecholamines Help Maintain Beating Activity in Neonatal Rat Cardiomyocyte Cultures
}

\author{
ARUNA R. NATARAJAN, QI RONG, ALEXANDER N. KATCHMAN, AND STEVEN N. EBERT \\ Department of Pediatrics [A.R.N.], Department of Pharmacology [Q.R., A.N.K., S.N.E.], Georgetown \\ University Medical Center, Washington, DC 20057, U.S.A.
}

\begin{abstract}
In the present study, we identify intrinsic cardiac adrenergic (ICA) cells in the neonatal rat heart using immunofluorescent histochemical staining techniques with antibodies that specifically recognize the major enzymes in the catecholamine biosynthetic pathway. ICA cells are most concentrated near the endocardial surface of ventricular myocardium, but are also found sporadically throughout the heart. In primary cultures of neonatal rat cardiomyocytes, ICA cells are closely associated with clusters of cardiomyocytes. To investigate a potential role for intrinsically produced catecholamines, we recorded beating rates in the presence and absence of the catecholamine-depleting agent reserpine or the adrenergic receptor blockers prazosin and timolol using videomicroscopy and photodiode sensors. Our results show that beating rates slow significantly when endogenous catecholamines are depleted or when their action is blocked with either a $\beta$ - or an $\alpha_{1}$-adrenergic receptor antagonist. These data
\end{abstract}

\section{ABSTRACT}

indicate that intrinsic cardiac catecholamines help to maintain beating rates in neonatal rat cardiomyocyte cultures via stimulation of $\alpha_{1}$ - and $\beta$-adrenergic receptors. This information should help to increase our understanding of the physiologic mechanisms governing cardiovascular function in neonates. (Pediatr Res 56: 411-417, 2004)

$\quad$ Abbreviations
ICA, intrinsic cardiac adrenergic
TH, tyrosine hydroxylase
DBH, dopamine $\beta$-hydroxylase
PNMT, phenylethanolamine $N$-methyltransferase
TRITC, tetramethylrhodamine isothiocyanate
DOPS, dihydroxyphenylserine
DMEM, Dulbecco's modified Eagle medium
HBSS, Hank's balanced salt solution

The catecholamines epinephrine and norepinephrine are neurotransmitter stress hormones that influence cardiac function profoundly. We and others have shown that the heart itself produces these catecholamines in specialized cells known as ICA cells (1-3). We have also shown that these cells are transiently and progressively associated with different regions of the embryonic rat heart that are important sites for pacemaking and conduction tissue development (e.g. sinoatrial node, atrioventricular canal, bundle of His, and ventricular septum) (3). ICA cells appear to persist in the heart postnatally as they have been found in neonatal $(1,4-6)$ and adult (1, 7-11) hearts. In adult hearts, ICA cells are often found clustered around sites of innervation $(6,7,10)$. In the neonate,

Received August 1, 2003; accepted April 26, 2004.

Correspondence: Steven N. Ebert, Ph.D., Department of Pharmacology, Georgetown University Medical Center, 3900 Reservoir Rd., NW, Washington, DC 20057, U.S.A.; e-mail: eberts@georgetown.edu

Supported in part by a grant to S.N.E. from the National Institutes of Health (R01HL58743). A.N. was supported by the Mentored Clinical Research Scholar Program Award, Grant RR17613, from the National Center for Research Resources, National Institutes of Health, Department of Health and Human Services.

DOI: 10.1203/01.PDR.0000136279.80897.4C sympathetic innervation of the heart is incomplete (12), and little is known about the specific distribution of ICA cells in the heart (13-15). Further, the functional significance of these ICA cells in the neonatal (or adult) heart is unknown.

The neonatal period is characterized by dramatic cardiovascular adaptation to extrauterine life. Although peripheral catecholamines are synthesized by the adrenal medulla (16-18) and organ of Zuckerkandl $(19,20)$ in the perinatal period, functional sympathetic innervation of the heart is not completed until the second week after birth in rats $(15,21)$. The neonatal rat heart, nevertheless, expresses fully functional $\alpha$ and $\beta$-adrenergic receptors, allowing for chronotropic responses to either $\alpha_{1}$ - or $\beta$-adrenergic agonists (22-24). Once functional sympathetic innervation of the heart is achieved, $\alpha_{1}$-agonists lose their ability to stimulate heart rate (22), which comes increasingly under $\beta$-adrenergic control, as seen in the adult $(25,26)$. Well before such innervation-dependent changes happen, however, chronotropic responses to exogenously administered catecholamines occur (27-31). The source(s) of endogenous catecholamines that regulate heart rate during perinatal development remain undefined. 
In the present study, we used immunofluorescence histochemical and cytochemical staining techniques to demonstrate the presence of ICA cells in neonatal rat hearts and primary cultures of cardiomyocytes isolated from neonatal rat hearts by their ability to express catecholamine biosynthetic enzymes. We also used pharmacological interventions to demonstrate possible roles for these locally produced catecholamines during cardiac development by evaluating beating rate responses in primary cultures of neonatal rat cardiomyocytes using videomicroscopy, photodiode sensors, and customized data acquisition/analysis software.

\section{METHODS}

Materials. All drugs and chemicals used for this study were purchased from Sigma Chemical (St. Louis, MO, U.S.A.). Drugs were prepared as concentrated stock solutions and frozen in small aliquots at $-80^{\circ} \mathrm{C}$. The stock aliquots were thawed immediately before use and were not refrozen. Rabbit anti-PNMT and anti-DBH antisera have been previously described (3). Mouse monoclonal anti-TH and anti-sarcomeric $\alpha$-actinin antibodies were purchased from Sigma Chemical. FITC-conjugated donkey anti-rabbit and TRITC-conjugated donkey anti-mouse secondary antibodies were obtained from Jackson Immunoresearch (West Grove, PA, U.S.A.).

Animals. Timed-pregnant Sprague-Dawley rats were obtained from Taconic Farms (Germantown, NY, U.S.A.). Neonatal pups were used within the first $2 \mathrm{~d}$ after birth. All experiments were conducted in strict concordance with the guidelines provided by the Georgetown University Animal Care and Use Committee and the National Institutes of Health.

Immunofluorescent histochemical and cytochemical staining. Double immunofluorescent staining of neonatal rat hearts and cardiomyocyte cultures was performed essentially as described previously (3). For immunofluorescent cytochemical staining of cultured cardiomyocytes, the myocytes were seeded onto collagen-coated coverslips (12 $\mathrm{mm}$ round) at the time of isolation, and allowed to grow in culture for 7-9 d before fixation. To fix the cells, the culture medium was removed and the cells were rinsed twice with PBS. The cells were then exposed to 4\% paraformaldehyde in PBS for $15 \mathrm{~min}$ at room temperature. At the end of this fixation period, the fixative was removed and the cells were washed twice with PBS. The cells were then used immediately for immunofluorescent staining or stored in PBS at $4^{\circ} \mathrm{C}$ for subsequent staining.

Because the anti-DBH and anti-PNMT antisera were produced in rabbits while the anti-TH antibody was produced in mice, FITC-conjugated anti-rabbit (green fluorescence) and TRITC-conjugated anti-mouse (red fluorescence) secondary antibodies were used to detect specific labeling of DBH- (or PNMT-) and TH-expressing cells, respectively. We have previously demonstrated the specificity of these antibodies using immunofluorescent histochemical staining assays (3).

Preparation of rat neonatal cardiomyocyte cultures. Neonatal rat cardiomyocytes were prepared according to Simpson and Savion (32), with the following modifications: Neonatal rat pups were killed by swift decapitation within the first $2 \mathrm{~d}$ after birth. The heart was immediately removed under aseptic con- ditions and placed in ice-cold sterile HBSS (no $\mathrm{Ca}^{++}$or $\mathrm{Mg}^{++}$). Enzymatic and mechanical dissociation of cardiomyocytes was then performed using the Neonatal Cardiomyocyte Isolation System supplied by Worthington Biochemicals (Freehold, NJ, U.S.A.). The hearts were minced on ice and digested overnight at $4^{\circ} \mathrm{C}$ with purified trypsin $(10 \mu \mathrm{g} / \mathrm{mL})$ in HBSS . On the following morning, the digested tissue was transferred to a $50-\mathrm{mL}$ conical tube and purified soybean trypsin inhibitor (40 $\mu \mathrm{g} / \mathrm{mL}$ ) was added to terminate trypsinization. The tissue was oxygenated and warmed to $37^{\circ} \mathrm{C}$. Purified collagenase $(10$ $\mathrm{U} / \mathrm{mL}$ ) was added and digestion proceeded for $45 \mathrm{~min}$ at $37^{\circ} \mathrm{C}$ with intermittent gentle swirling. Mild trituration was then used to mechanically dissociate the digested tissue, and singlecell suspensions were obtained by filtering this digested material through $70 \mu \mathrm{m}$ sterile mesh filters. The cells were collected by low-speed centrifugation. The supernatant was discarded and the cell pellet resuspended in DMEM (high glucose, Biofluids, Rockville, MD, U.S.A.) culture medium containing $10 \%$ defined iron-supplemented bovine calf serum (Hyclone Laboratories, Logan, UT, U.S.A.), $2 \mathrm{mM}$ glutamine, and gentamicin sulfate $(50 \mu \mathrm{g} / \mathrm{mL})$. In some experiments, the serum was pretreated with charcoal before adding it to the media ( $10 \%$ volume as above) to remove possible trace amounts of catecholamines that may have been present. The cells were then "preplated" to remove fibroblasts as described previously (7), and the remaining cells counted and seeded onto multiwell culture plates at a density of 75,000 cells per $\mathrm{cm}^{2}$. The media was changed every $2-3 \mathrm{~d}$ beginning the day after seeding. Bromodeoxyuridine $(0.1 \mathrm{mM})$ was added to the culture media for the first $3 \mathrm{~d}$ to further minimize contamination from fibroblasts (32).

Beating rate measurements. Beating activity of cultured cardiomyocytes was measured by edge-detection using photodiode sensors attached to a monitor screen depicting beating cells viewed by videomicroscopy. The entire microscope was enclosed in a temperature-controlled $\left(37^{\circ} \mathrm{C}\right)$, custom-designed incubation chamber (Part numbers: 8400-005, 8506-050, and 8537-025; Coy Laboratory Products, Inc., Grass Lake, MI, U.S.A.) fed with $5 \% \mathrm{CO}_{2} / 95 \%$ air to maintain constant $\mathrm{pH}$. The photodiode sensor signal was filtered with an 8-pole Bessel low-pass filter (Model 900CT, Frequency Devices, Inc., Havervill, MA, U.S.A.) with a cutoff frequency of $1000 \mathrm{~Hz}$, and relayed to a PC equipped with a National Instruments data acquisition board and LabView 4.0 processing software. Parallel outputs were also directed to a Gould TA550 chart recorder and an oscilloscope. Data were continuously acquired and stored to disk for later analysis. Drugs were added and immediately mixed in the media overlying the cells in each well. Predrug (baseline) measurements were performed first, and the indicated drug(s) were added as a $100 \times$ stock to the preexisting media on each well, and rapidly mixed. Beating rates were remeasured within 2-3 min after drug addition.

Data analysis. The number of beating rate measurements was typically obtained from different wells of multi-well plates (usually six wells per plate) seeded from the same preparation of myocytes. Measurements were obtained immediately before and after the addition of specified drugs. In the reserpine experiment, beating rate measurements were obtained at mul- 
tiple time-points after drug administration. Each experiment was replicated at least twice using independently isolated cultures, and a representative result from each is shown. Unless otherwise indicated, all beating rate measurements were performed on cells that had been cultured for 7-9 d. All data are expressed as mean \pm SEM. Statistical significance was assessed by one-way ANOVA, with $p<0.05$ required to reject the null hypothesis. Posthoc testing was performed using Bonferroni's correction for multiple comparisons.

\section{RESULTS}

To identify ICA cells in neonatal rat hearts, we used immunofluorescent histochemical staining techniques using antibodies that specifically recognize the major catecholamine biosynthetic enzymes. Many brightly labeled fluorescent cells were identified using anti-PNMT antiserum (Fig. 1, $A$ and $C$, arrows). Co-staining with an anti-TH antibody revealed an essentially identical pattern of expression (Fig. 1, $B$ and $D$, arrows; compare with $A$ and $C$, respectively). These ICA cells were primarily found in the ventricular myocardium near the endocardial surface, but were also observed sporadically throughout the heart. For anatomical reference, the ventricular lumen (VL) is indicated (Figs. 1 and 2). A similar pattern of co-staining was observed when we used anti-DBH and anti-TH antibodies (Fig. 2). Positively labeled cells appeared bright, were roundish or triangular in shape, and primarily displayed cytoplasmic staining patterns. This was most evident at higher magnification (Figs. 1 and 2, panels $C$ and $D$ ). Thus, each of the three independent catecholamine biosynthetic enzyme $(\mathrm{TH}$,
PNMT
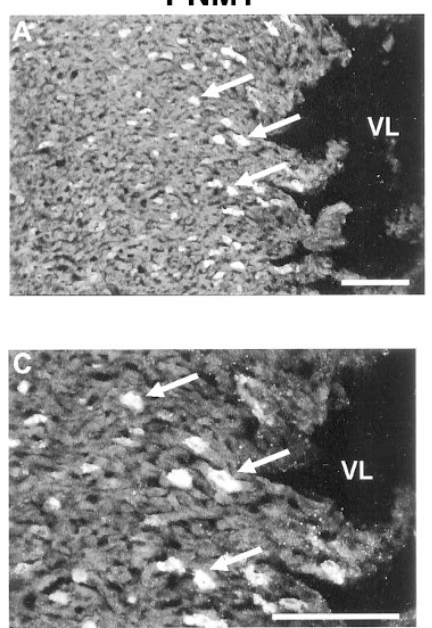

TH
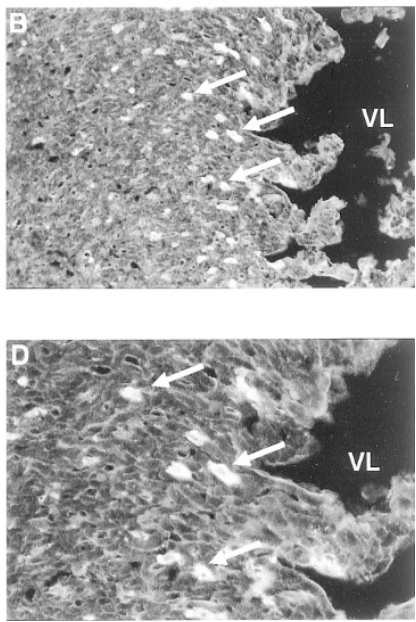

Figure 1. Immunofluorescent histochemical staining of neonatal rat ventricular myocardium for PNMT and TH. Neonatal rat sections (10 $\mu \mathrm{m}$ thickness) were incubated with rabbit anti-PNMT antiserum and mouse anti-TH ascites. To detect specific staining, FITC-conjugated anti-rabbit IgG and TRITCconjugated anti-mouse IgG were subsequently reacted with the sections. ( $A$ and $B)$ Low-magnification ( $20 \times$ objective) images of the same section visualized through FITC- and TRITC-specific filters, respectively. ( $C$ and $D$ ) Highmagnification $(40 \times$ objective) images of the same section visualized through FITC- and TRITC-specific filters, respectively. Arrows identify a subset of cells that were co-stained with the anti-PNMT and anti-TH antibodies. No "bleed-through" fluorescence was observed when either one of the primary antibodies was omitted (data not shown), as previously demonstrated (10). Scale bars, $100 \mu \mathrm{m}$.
DBH
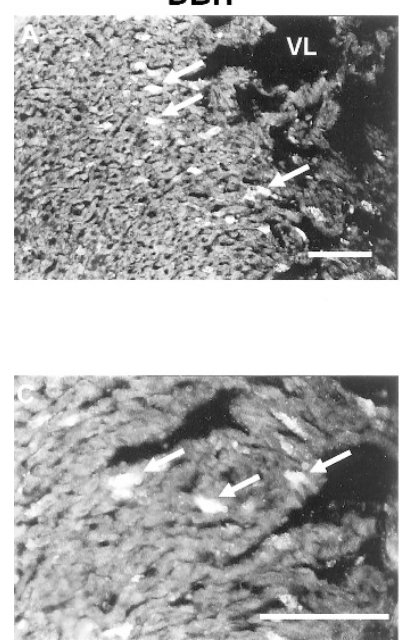

TH

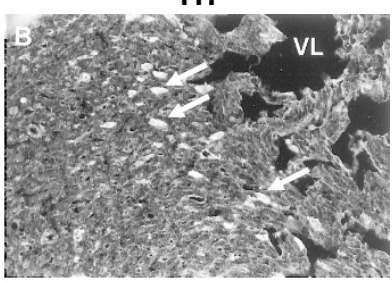

Figure 2. Immunofluorescent histochemical staining of neonatal rat ventricular myocardium for DBH and TH. Neonatal rat sections (10 $\mu \mathrm{m}$ thickness) were incubated with rabbit anti-DBH antiserum and mouse anti-TH ascites. To detect specific staining, FITC-conjugated anti-rabbit IgG and TRITCconjugated anti-mouse IgG were subsequently reacted with the sections. ( $A$ and $B)$ Low-magnification $(20 \times$ objective $)$ images of the same section visualized through FITC- and TRITC-specific filters, respectively. Arrows identify a subset of cells that were co-stained with the anti-DBH and anti-TH antibodies. $(C$ and $D)$ High-magnification ( $40 \times$ objective) images of a different region of the same section visualized through FITC- and TRITC-specific filters, respectively. Arrows identify a subset of cells that were co-stained with the anti-DBH and anti-TH antibodies. No "bleed-through" fluorescence was observed when either one of the primary antibodies was omitted (data not shown), as previously demonstrated (10). Scale bars, $100 \mu \mathrm{m}$.

DBH, and PNMT) antibodies selectively labeled a highly similar subset of cardiac cells in neonatal rat hearts.

To view these presumptive ICA cells in relation to cardiomyocytes, we co-stained sections for PNMT and a myocytespecific marker, sarcomeric $\alpha$-actinin. As shown in Figure $3 A$, individual ICA cells were again identified in ventricular myo-
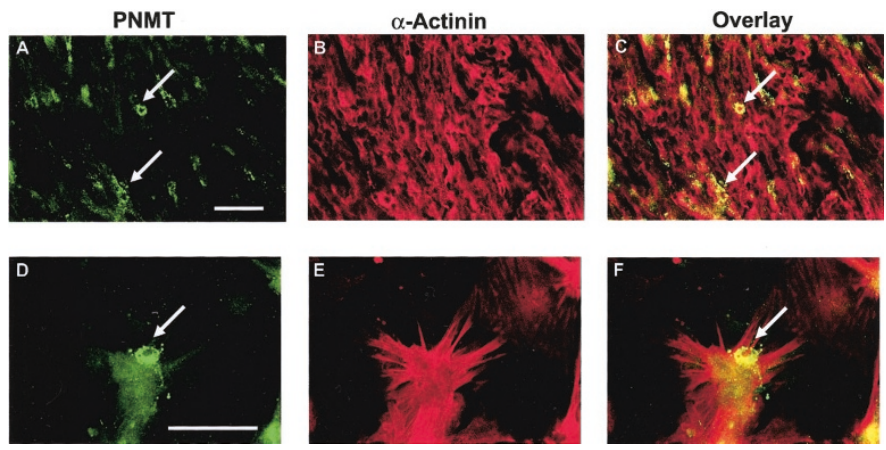

Figure 3. Dual immunocytochemical fluorescent staining for PNMT and sarcomeric $\alpha$-actinin in neonatal rat sections and cultured cardiomyocytes. $(A-C)$ Neonatal rat ventricular myocardial section. $(D-F)$ Cultured neonatal rat cardiomyocytes ( $7 \mathrm{~d}$ in culture). Fixed tissue sections and cells were reacted with rabbit anti-PNMT antiserum and mouse anti-sarcomeric $\alpha$-actinin ascites fluid. Specific labeling was detected using fluorescence microscopy after reaction with FITC-conjugated anti-rabbit IgG and TRITC-conjugated antimouse IgG. Arrows indicate examples of cells that were positively stained for PNMT. Images were captured using either the FITC-specific ( $A$ and $D$ ) or the TRITC-specific ( $B$ and $E$ ) filter. The "Overlay" images depicted in panels $C$ and $F$ were obtained by combining panels $A$ and $B$, or $C$ and $D$, respectively, using Adobe Photoshop 5.5 software. Scale bars, $100 \mu \mathrm{m}$. 
cardium. When co-stained for $\alpha$-actinin, a characteristic ladder-like sarcomeric pattern was evident in many myocytes (Fig. $3 B$ ). By superimposing the PNMT and $\alpha$-actinin images, we obtained an "overlay" image where ICA cells can be visualized in direct juxtaposition to underlying myocytes (Fig. $3 C)$.

To identify ICA cells in primary cultures of neonatal rat cardiomyocytes, we seeded the cardiomyocytes onto collagencoated coverslips and allowed them to develop into synchronous rhythmic beating clusters of cardiomyocytes over a 7-d period. The cells were then fixed and co-immunofluorescently labeled for PNMT and sarcomeric $\alpha$-actinin (Fig. 3, $D$ and $E$, respectively). PNMT staining was punctate and cytoplasmic, whereas $\alpha$-actinin staining was evident in sarcomeric structures (Fig. $3 E$ ). The overlay image shown in Figure $3 F$ clearly shows an ICA cell sitting atop a cluster of cardiomyocytes. ICA cells could also be identified in these cultures by co-staining for $\mathrm{DBH}$ and TH (not shown). Although the relative abundance of ICA cells in these neonatal rat cardiomyocyte cultures was low (only $\sim 1 \mathrm{ICA}$ cell/ $2 \times 10^{5}$ myocytes), these results nevertheless show that ICA cells were present and that they were closely associated with cardiomyocyte clusters.

To determine whether beating activity in culture was dependent upon local catecholamine production, the wells were treated with increasing concentrations of reserpine, a catecholamine-depleting agent. Significant dose-dependent decreases in beating rates were observed in the presence of reserpine (Fig. 4, $A$ and $B$ ). These decreases could be largely reversed by the addition of norepinephrine (Fig. 4C). These results suggest that endogenous catecholamines are needed for the maintenance of beating rates in these cardiomyocyte cultures.

Consistent with this idea, acute treatment of spontaneously beating myocyte cultures with either the $\alpha_{1}$-selective antagonist, prazosin, or the $\beta$-selective antagonist, timolol, led to dose-dependent decreases in the beating rate within seconds after drug application (Fig. 5). Significant slowing ( 25\% decrease) in the beating rate was observed in the presence of 1 $\mu \mathrm{M}$ prazosin $(p<0.01, n=10)$. When the prazosin concentration was increased to $10 \mu \mathrm{M}$, severe depression $(>85 \%$ decrease) in the beating rate occurred. Timolol also caused significant concentration-dependent slowing in the beating rate $(p<0.01, n=6)$. These results show that application of either $\alpha_{1}$ - or $\beta$-adrenergic receptor blockers lead to concentrationdependent slowing of beating rate.

To test the possibility that serum catecholamines may have influenced our results, we stripped the serum using charcoaltreatment to remove any trace amounts of catecholamines that may have been present. Beating activity was maintained for at least $72 \mathrm{~h}$ after charcoal-stripping (Fig. 6). The $\beta$-blocker timolol strongly suppressed beating activity under these conditions, an effect that was fully rescued by the addition of epinephrine (Fig. 6). Similarly, application of the $\alpha_{1}$-blocker prazosin led to significant decreases in beating rates (Fig. 7). The addition of the $\alpha_{1}$-selective agonist, L-phenylephrine, resulted in a dose-dependent reversal of the prazosin-induced decrease in beating rate. These results show that $\alpha_{1}$ - and $\beta$-adrenergic receptor antagonists were effective at blocking beating even in the absence of any potential influence from serum catecholamines.

\section{DISCUSSION}

The results presented in this study indicate that intrinsic cardiac catecholamines play an important role in helping to maintain beating rates of cultured neonatal rat cardiomyocytes. The evidence supporting this claim is summarized as follows: i) using immunofluorescent staining techniques, ICA cells were found to be present in close association with cardiomyocytes both in vivo and in vitro; ii) depletion of endogenous catecholamines by treatment with reserpine led to significant decreases in beating rate that were subsequently recovered by the addition of norepinephrine; iii) blockade of either $\alpha_{1}$ - or $\beta$-adrenergic receptors led to concentration-dependent decreases in beating rates that could be "rescued" by the addition of adrenergic agonists; and iv) elimination of potential trace amounts of catecholamines from the serum used for culturing the cardiomyocytes did not prevent either the $\beta$-antagonist, timolol, or the $\alpha_{1}$-antagonist, prazosin, from causing significant decreases in the beating rate. Although it has been known for many years that exogenous catecholamines can influence cardiovascular function $(27,33,34)$, this is the first report to systematically demonstrate that endogenous cardiac catecholamines, acting through $\alpha_{1}$ - and $\beta$-adrenergic receptors, are necessary for the maintenance of beating activity in neonatal cardiomyocyte cultures.

As shown in the present report, the adrenergic antagonists used in this study were effective at slowing "spontaneous" beating rates even when charcoal-stripped serum, free of catecholamines, was used. In general, serum was included in our media because the cells appeared healthier and beating activity was more stable when serum (nonstripped) was used as a media supplement. When charcoal-stripped or serum-free media was used, the concentrations of the antagonists or reserpine required to inhibit beating activity was much less than that needed in the presence of untreated serum. This difference in drug sensitivity may reflect a decrease in nonspecific drug binding by serum proteins, and, hence, lower concentrations of the drugs were needed to produce the same effect as when nonstripped serum was used. It is also possible that other small molecules and growth factors removed by the charcoalstripping procedure could have rendered the cells more sensitive to drugs that deplete or block catecholamine actions. We submit, however, that the possibility that the serum was a significant source of catecholamines is highly unlikely because we found no measurable amounts of either epinephrine or norepinephrine using a sensitive RIA (lower limit of assay sensitivity $\sim 4 \mathrm{pg} / \mathrm{mg}$ total protein $/ \mathrm{mL}$ ) (3). We also performed additional experiments using defined, serum-free media (22, $35)$ where we found that reserpine $(1 \mu \mathrm{M})$ was still effective at blocking beating activity (data not shown). As was the case when serum was included (Fig. 4), the cessation of beating activity in the presence of reserpine could be recovered by the addition of epinephrine or norepinephrine. Taken together, these results suggest that intrinsically produced catecholamines function to regulate beating activity in cardiomyocyte cultures 

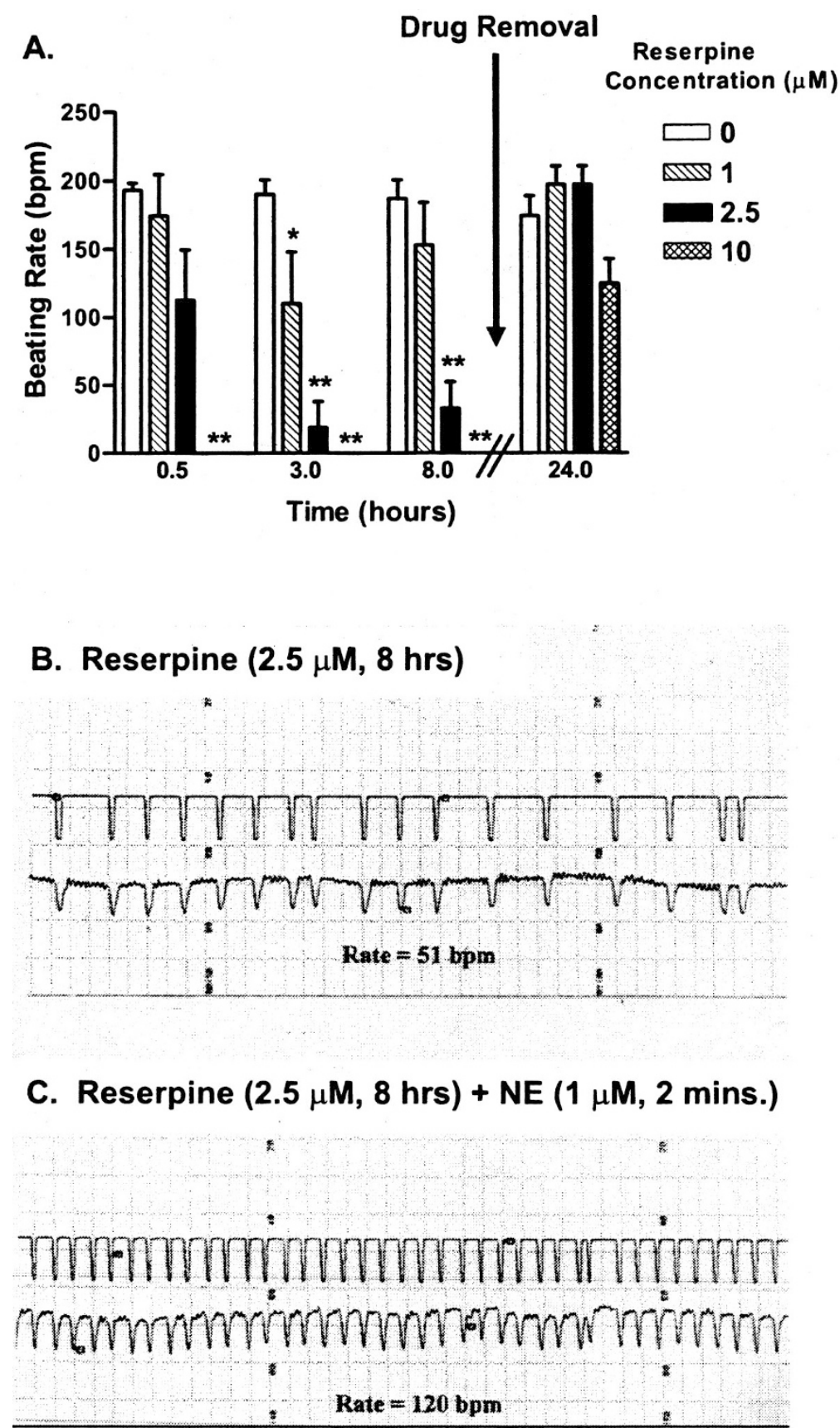

Figure 4. Effect of the catecholamine-depleting agent reserpine on the maintenance of beating rate in neonatal rat cardiomyocyte cultures. Spontaneously beating neonatal rat cardiomyocytes were allowed to develop for a period of $7 \mathrm{~d}$ in culture, and different concentrations of reserpine were added directly to the culture media in each well, as indicated. (A) Beating rates were measured at the indicated time points in the presence of the indicated concentrations of reserpine ( $n=6$ /group). After the measurement at the 8-h time point, the reserpine-containing media was removed (indicated by arrow with designation, "Drug Removal"), the cells were rinsed with drug-free media, and replenished with drug-free growth media. Beating rate measurements were then re-evaluated at the 24-h time point. $(B)$ Sample recording of beating activity after $8 \mathrm{~h}$ of reserpine $(2.5 \mu \mathrm{M})$ treatment in one well. $(C)$ Effect of norepinephrine $(N E, 1 \mu \mathrm{M})$ on the beating activity of the same cells recorded in panel $B$ approximately $2 \mathrm{~min}$ after the addition of NE (before removal of reserpine). Upper trace in panels $B$ and $C$ represents the "event detector signal" used to measure rate. Lower trace in same panels represents the actual photodiode signal. $*^{*} p 0.05 ; * * p<0.01$.

and that there was little or no contribution of catecholamines from the serum.

Our results are consistent with those published by Huang et al. (1), who first identified ICA cells in fetal human hearts. Using independently generated anti- $\mathrm{TH}$, anti- $\mathrm{DBH}$, and antiPNMT antibodies, both we (3) and Huang et al. have shown that ICA cells are present in fetal and neonatal hearts. We have further shown that ICA cells are present in close association with clusters of cardiomyocytes in primary culture. Although ICA cells and myocytes may be derived from common progenitor cells (3), no detectable expression of the myocyte marker protein, sarcomeric $\alpha$-actinin, was observed in ICA cells at the neonatal stage of development. The lack of costaining for sarcomeric $\alpha$-actinin suggests that ICA cells do not have sarcomeres. Indeed, their shape helps to distinguish ICA cells from their nearby myocyte neighbors. Whereas myocytes 

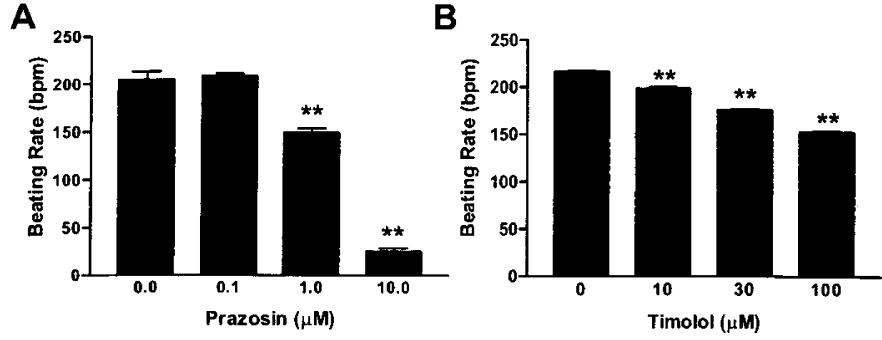

Figure 5. Effects of adrenergic antagonists on beating rates in cultured neonatal rat cardiomyocytes. Cardiomyocytes were allowed to develop spontaneous beating activity over a period of $7 \mathrm{~d}$ before the addition of drugs. Each drug was added at the indicated concentrations and beating rates were assessed within 1-2 min after drug addition. (A) Effect of increasing concentrations of the $\alpha_{1}$-selective antagonist prazosin. ( $\left.B\right)$ Effect of increasing concentrations of the $\beta$-selective antagonist timolol. ${ }^{* *} p<0.01$ ( $n=6$ /group).

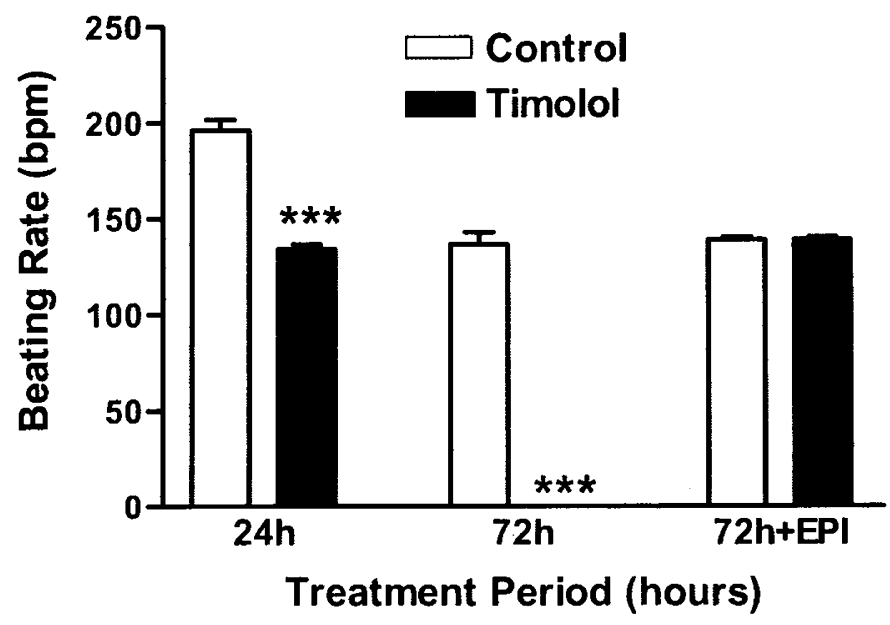

Figure 6. Effect of $\beta$-adrenergic blockade on beating rates in neonatal rat cardiomyocytes cultured using media containing charcoal-stripped serum. Neonatal rat cardiomyocytes were allowed to develop spontaneous beating activity under normal growth conditions for a period of $7 \mathrm{~d}$. On $\mathrm{d} 7$, the culture media was replaced with media containing charcoal-stripped serum to remove any residual catecholamines that may have been present in the serum. Timolol $(10 \mu \mathrm{M})$ was added to the culture medium containing the charcoal-stripped serum on half of the cultures at the time of media change on $\mathrm{d} 7$. Beating rates were then assessed at 24 and $72 \mathrm{~h}$ after drug treatment (cells were cultured in the continuous presence of timolol). Addition of epinephrine $(1 \mu \mathrm{M})$ enabled full recovery of beating rate for at least $30-60 \mathrm{~min} .{ }^{* * *} p<0.001$ relative to control ( $n=6$ /group).

tend to be rectangular in appearance, ICA cells were somewhat roundish or triangular in shape, consistent with previous findings (1).

Neonatal rat cardiomyocyte cultures are a well-established model for studying the functional development of cardiac beating activity $(32,35)$, and early studies have shown that either $\alpha_{1}$ - or $\beta$-agonists could stimulate beating activity in these cultures (22). Once sympathetic innervation is achieved, the chronotropic effects of $\alpha_{1}$-agonists subside in favor of the more adult-like $\beta$-adrenergic-mediated control of heart rate (24). During the early neonatal period, however, there is minimal sympathetic nerve influence. Intrinsic cardiac adrenergic cells may, therefore, play a more prominent role in the heart during this early postnatal period of development.

High demands are placed on cardiovascular function as newborns adapt to extrauterine life. Having a local source of

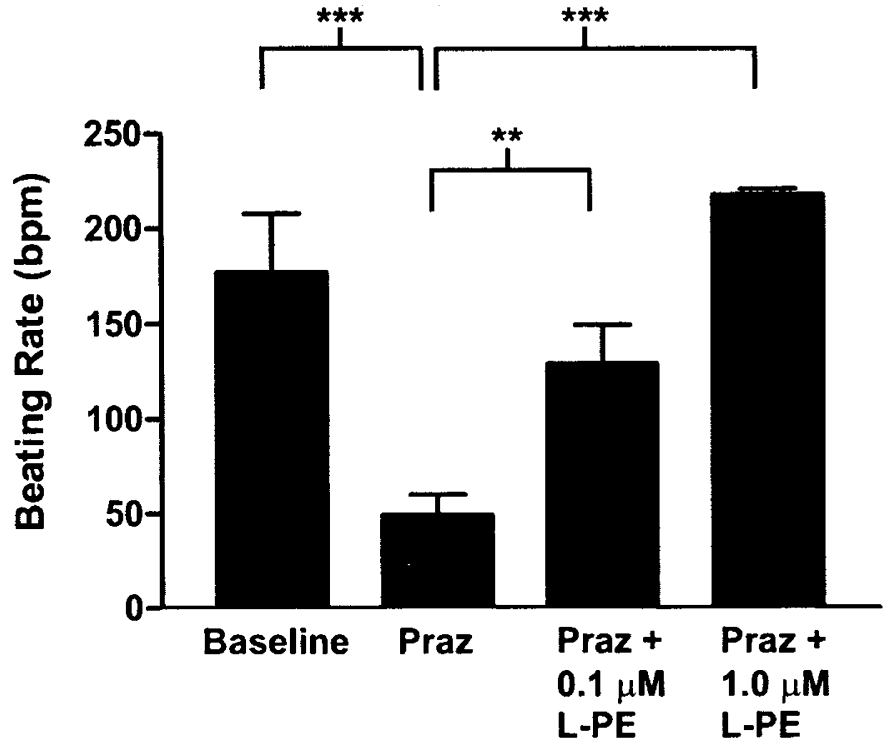

Figure 7. Effect of $\alpha_{1}$-adrenergic blockade on the beating rates in neonatal rat cardiomyocytes cultured in media containing charcoal-stripped serum. Application of the $\alpha_{1}$-antagonist, prazosin $(1 \mu \mathrm{M})$ resulted in a significant reduction in the beating rate compared with baseline control. This reduction in the beating rate was reversed by the addition of the $\alpha_{1}$-agonist, L-phenylephrine (L-PE) at the indicated concentrations. $* * p<0.01$; ***p $<0.001$ ( $n=$ 12/group).

catecholamines within the heart may enhance cardiovascular function, though this may not be absolutely necessary for survival because certain mice and humans who lack the ability to produce norepinephrine and epinephrine due to a genetic disruption of the gene encoding DBH have survived past the early postnatal period $(36,37)$. In mice, most $d b h^{-1-}$ embryos die from cardiovascular failure in utero, but can be "rescued" by administration of DOPS in the maternal drinking water (37). DOPS is a synthetic compound that can be converted into norepinephrine in vivo via a DBH-independent mechanism. Once born, some DOPS-rescued $d b h^{-1-}$ embryos are then able to survive without further drug interventions, though it is unclear how much DOPS and norepinephrine/epinephrine may persist after birth in the neonate. Nevertheless, it is clear that norepinephrine and/or epinephrine are important for postnatal survival since "about $40 \%$ of the mutant $\left(d b h^{-1-}\right)$ mice born die within several days of birth and another $20 \%$ die during the third, fourth, and fifth postnatal weeks" (38).

What are the source(s) of endogenous catecholamines? We have shown that neonatal rat cardiomyocyte cultures have the capacity to synthesize catecholamines. Further, we demonstrated a possible role for intrinsic cardiac catecholamines in regulating beating rates. These findings are consistent with the idea proposed by Hsu (34) more than $70 \mathrm{y}$ ago when he noted strong cardiac responses upon applying epinephrine or acetylcholine to embryonic chick hearts: "Should it be possible, however, to detect the presence of acetylcholine and adrenaline in the embryo of the age we have worked with, our result would indicate a possible humoral control of heart rate before the inception of nervous control." Thus, our results suggest that ICA cells serve as an important "humoral" source of adrenergic 
hormones that function to regulate beating activity before maturation of sympathetic innervation.

\section{REFERENCES}

1. Huang MH, Friend DS, Sunday ME, Singh K, Haley K, Austen KF, Kelly RA, Smith TW 1996 An intrinsic adrenergic system in mammalian heart. J Clin Invest 98:12981303

2. Ebert SN, Baden JM, Mathers LH, Siddall BJ, Wong DL 1996 Expression of phenylethanolamine $\mathrm{N}$-methyltransferase in the embryonic rat heart. $\mathrm{J}$ Mol Cell Cardiol 28:1653-1658

3. Ebert SN, Thompson RP 2001 Embryonic epinephrine synthesis in the rat heart before innervation: association with pacemaking and conduction tissue development. Circ Res 88:117-124

4. Ignarro LJ, Shideman FE 1968 Appearance and concentrations of catecholamines and their biosynthesis in the embryonic and developing chick. J Pharmacol Exp Ther 159:38-48

5. Papka RE 1974 A study of catecholamine-containing cells in the hearts of fetal and postnatal rabbits by fluorescence and electron microscopy. Cell Tissue Res 154:471484

6. Padbury JF, Diakomanolis ES, Lam RW, Hobel CJ, Fisher DA 1981 Ontogenesis of tissue catecholamines in fetal and neonatal rabbits. J Dev Physiol 3:297-303

7. Jacobowitz D 1967 Histochemical studies of the relationship of chromaffin cells and adrenergic nerve fibers to the cardiac ganglia of several species. J Pharmacol Exp Ther 158:227-240

8. Ellison JP, Hibbs RG 1974 Catecholamine-containing cells of the guinea pig heart: an ultrastructural study. J Mol Cell Cardiol 6:17-26

9. Spurgeon HA, Priola DV, Montoya P, Weiss GK, Alter WA 3rd 1974 Catecholamines associated with conductile and contractile myocardium of normal and denervated dog hearts. J Pharmacol Exp Ther 190:466-471

10. Cottle MK 1984 Distribution of catecholamine-containing cells in the atrial region in rats and ground squirrels. Histochem J 16:1137-1146

11. Dail WG, Palmer GC 1973 Localization and correlation of catecholamine-containing cells with adenyl cyclase and phosphodiesterase activities in the human fetal heart. Anat Rec 177:265-287

12. De Champlain J, Malmfors T, Olson L, Sachs C 1970 Ontogenesis of periphera adrenergic neurons in the rat: pre- and postnatal observations. Acta Physiol Scand 80:276-288

13. Bareis DL, Morgan RE, Lau C, Slotkin TA 1981 Maturation of sympathetic neurotransmission in the rat heart. IV. Effects guanethidine-induced sympathectomy on neonatal development of synaptic vesicles, synaptic terminal function and heart growth. Dev Neurosci 4:15-24

14. Deskin R, Slotkin TA 1981 Central catecholaminergic lesions in the developing rat: effects of cardiac noradrenaline levels, turnover and release. J Auton Pharmaco $1: 205-210$

15. Lau C, Burke SP, Slotkin TA 1982 Maturation of sympathetic neurotransmission in the rat heart. IX. Development of transsynaptic regulation of cardiac adrenergic sensitivity. J Pharmacol Exp Ther 223:675-680

16. Kvetnansky R, Jahnova E, Torda T, Strbak V, Balaz V, Macho L 1978 Changes of adrenal catecholamines and their synthesizing enzymes during ontogenesis and aging in rats. Mech Ageing Dev 7:209-216

17. Verhofstad AA, Hokfelt T, Goldstein M, Steinbusch HW, Joosten HW 1979 Appearance of tyrosine hydroxylase, aromatic amino-acid decarboxylase, dopamine betahydroxylase and phenylethanolamine $\mathrm{N}$-methyltransferase during the ontogenesis of the adrenal medulla: an immunohistochemical study in the rat. Cell Tissue Res 200:1-13
18. Coupland RE, Tomlinson A 1989 The development and maturation of adrenal medullary chromaffin cells of the rat in vivo: a descriptive and quantitative study. Int J Dev Neurosci 7:419-438

19. Peters LL, Wood BG 1987 The prenatal development of the organ of Zuckerkandl in rats. Life Sci 41:1355-1359

20. Tian H, Hammer RE, Matsumoto AM, Russell DW, McKnight SL 1998 The hypoxia-responsive transcription factor EPAS1 is essential for catecholamine homeostasis and protection against heart failure during embryonic development. Genes Dev 12:3320-3324

21. Nyquist-Battie C, Cochran PK, Sands SA, Chronwall BM 1994 Development of neuropeptide $\mathrm{Y}$ and tyrosine hydroxylase immunoreactive innervation in postnatal rat heart. Peptides 15:1461-1469

22. Simpson P 1985 Stimulation of hypertrophy of cultured neonatal rat heart cells through an alpha 1-adrenergic receptor and induction of beating through an alpha 1and beta 1-adrenergic receptor interaction. Evidence for independent regulation of growth and beating. Circ Res 56:884-894

23. Shigenobu K, Tanaka H, Kasuya Y 1988 Changes in sensitivity of rat heart to norepinephrine and isoproterenol during pre- and postnatal development and its relation to sympathetic innervation. Dev Pharmacol Ther 11:226-236

24. Drugge ED, Rosen MR, Robinson RB 1985 Neuronal regulation of the development of the alpha-adrenergic chronotropic response in the rat heart. Circ Res 57:415-423

25. Hou QC, Seidler FJ, Slotkin TA 1989 Development of the linkage of beta-adrenergic receptors to cardiac hypertrophy and heart rate control: neonatal sympathectomy with 6- hydroxydopamine. J Dev Physiol 11:305-311

26. Steinberg SF, Rosen TS, Malfatto G, Rosen MR 1991 Beta adrenergic modulation of cardiac rhythm in a rat model of altered sympathetic neural development. J Mol Cell Cardiol 23(suppl 1):47-52

27. Hall E 1957 Acetylcholine and epinephrine effects on the embryonic rat heart. J Cell Physiol 49:187-200

28. Robkin MA, Shepard TH, Dyer DC, Guntheroth WG 1976 Autonomic receptors of the early rat embryo heart growth and development. Proc Soc Exp Biol Med 151:799-803

29. Pappano AJ 1977 Ontogenetic development of autonomic neuroeffector transmission and transmitter reactivity in embryonic and fetal hearts. Pharmacol Rev 29:3-33

30. Wildenthal K 1973 Maturation of responsiveness to cardioactive drugs. Differential effects of acetylcholine, norepinephrine, theophylline, tyramine, glucagon, and dibutyryl cyclic AMP on atrial rate in hearts of fetal mice. J Clin Invest 52:2250-2258

31. Papp JG 1991 Cardiac responses to drugs at the extremes of age. Therapie 46:283291

32. Simpson P, Savion S 1982 Differentiation of rat myocytes in single cell cultures with and without proliferating nonmyocardial cells. Cross-striations, ultrastructure, and chronotropic response to isoproterenol. Circ Res 50:101-116

33. Markowitz C 1931 Response of explanted embryonic cardiac tissue to epinephrine and acetylcholine. Am J Physiol 97:271-275

34. Hsu F-Y 1933 The effect of adrenaline and acetylcholine on the heart rate of the chick embryo. Chin J Physiol VII:243-252

35. Simpson P, McGrath A, Savion S 1982 Myocyte hypertrophy in neonatal rat heart cultures and its regulation by serum and by catecholamines. Circ Res 51:787-80

36. Robertson D, Haile V, Perry SE, Robertson RM, Phillips JA 3rd, Biaggioni I 1991 Dopamine beta-hydroxylase deficiency. A genetic disorder of cardiovascular regulation. Hypertension 18:1-8

37. Thomas SA, Matsumoto AM, Palmiter RD 1995 Noradrenaline is essential for mouse fetal development. Nature 374:643-646

38. Thomas SA, Palmiter RD 1998 Examining adrenergic roles in development, physiology, and behavior through targeted disruption of the mouse dopamine betahydroxylase gene. Adv Pharmacol 42:57-60 